\title{
Diseño de la arquitectura pública con identidad cultural indígena Pewenche en la localidad de Ralco, Alto Biobío
}

\author{
Pewenche Indigenous Cultural Identity in the \\ Design of Public Buildings in Ralco, Alto Biobío
}

\section{${ }^{1}$ Felipe Quezada Vidal}

\begin{abstract}
RESUMEN
Esta investigación nace a partir del interés que se ha comenzado a desarrollar en la zona de Alto Biobío, debido a su creación como comuna con identificación Pewenche. El estudio contrasta el análisis de cómo criterios de diseño arquitectónico indígena pertinente son aplicados en edificios de equipamiento público en la localidad de Ralco, con el nivel de pertenencia que presentan estos edificios, medido en una encuesta a la población local, y, en consecuencia, de la imagen urbana con identidad cultural que busca proyectar Ralco a través de ellos. Se seleccionan cinco criterios de diseño pertinente: orientación, morfología, adaptación a clima, materialidad y participación en el diseño, los cuales son utilizados como bases al momento de realizar los análisis y comparaciones del diseño de los edificios seleccionados como muestra de este estudio exploratorio (Museo Pehuenche y edificio de la Municipalidad), con la medición de la importancia y la pertenencia de estos edificios recogido en la encuesta. Se genera un cruce de información entre los criterios de diseño apropiado aplicados en los edificios de equipamiento público, arrojando los resultados sobre la representatividad de estos y refinando los lineamientos bases que servirán como insumos de diseño para futuras proyecciones de edificios públicos con pertenencia cultural Pewenche.
\end{abstract}

Palabras clave

Pewenche, Identidad Indígena, Pertenencia Cultural, Equipamiento Público

\begin{abstract}
This research springs from the interest in the area of Alto Biobio due to its recent creation as a municipality and its strong cultural identity with the Pewenche people. The study contrasts how relevant Indigenous architectural design criteria are applied in buildings of public facilities in the town of Ralco, with the level of belonging as gathered from a survey of the local population, and consequently, the culturally relevant urban image that Ralco seeks to project through these buildings. For this reason, five relevant design criteria were selected - orientation, morphology and adaptation to climate and through materiality, and participation in the design process - to analyze the design of public buildings selected as samples for this exploratory study (Pewenche Museum and Municipal Building). Finally, the contrast between the relevant design criteria and the actual representativeness of the buildings serves to refine basic guidelines that will serve as design inputs for future projects of Pewenche culturally relevant public buildings.
\end{abstract}

\section{Keywords}

Pewenche, Indigenous Identity, Cultural Belonging, Public Buildings 


\section{INTRODUCCIÓN}

"Un pueblo que se aferra al Pehuén y convive con la Nehuen Mapu Dewiñ merece respeto y reconocimiento" (Nicolasa Quintremán, citada en Toro, 2013).

La presente investigación estudia la arquitectura pública con pertenencia cultural indígena, analizando si edificios de equipamiento público ubicados en Ralco -región del Biobío, comuna de Alto Biobío, al sur de Chile- logran interpretar la identidad Pewenche mediante criterios de diseño arquitectónico recogidos de la literatura. De acuerdo con Díaz (2015), los edificios públicos de Ralco -inserto, como está este espacio, en un territorio indígena que históricamente ha sido despojado de tierras e identidad (Huiliñir, 2015)- son los grandes responsables de exponer al territorio, reconociendo una cultura y proyectando una identidad territorial.

Alto Biobío es una comuna reciente, independiente desde el año 2004, en la cual el 70\% de la población se declara Pewenche. Es decir, unas 6.200 personas aproximadamente (PLADECO, 2014). Esta fue la razón principal de su autonomía comunal. Dentro de esta, se encuentra la localidad de Ralco, que es el centro urbano de la comuna, donde se albergan sus servicios y edificios de equipamiento público. El alcalde de la comuna, Nivaldo Piñaleo Llaulén, plantea que Ralco presenta el doble desafío del desarrollo urbano y de la integración cultural Pewenche. Por esto, se habrian hecho grandes esfuerzos por entregar una imagen que refleje la identidad territorial mediante sus edificios públicos (Araos, 2012).

En esa línea, la comuna de Alto Biobío se presenta como un caso interesante de estudio porque manifiesta una identidad cultural bien definida. Identidad aquí se refiere al sentimiento de pertenencia de la población hacia un territorio (Huiliñir, 2015). De esta manera, lo que importa para el presente estudio es que la identidad del centro urbano de Ralco está en construcción, como área urbana recientemente creada y como lugar de encuentro intercultural donde coexisten poblaciones y no indígenas. Así, el concepto interculturalidad es clave para el desarrollo urbano de Ralco, ya que busca reflejar una pertenencia cultural mediante la pertinencia del diseño de sus edificios públicos. Esa pertinencia, es definida por Díaz (2015) desde la arquitectura como una reinterpretación adecuada de las principales características de un pueblo o comunidad. De esa manera, el diseño pertinente intercultural no busca solo repetir criterios de construcciones ancestrales (como la ruka), sino crear sentimientos de pertenencia en un contexto nuevo.

La hipótesis propuesta aquí es que los edificios de equipamiento público interculturales de Ralco recogen algunos criterios de diseño pertinentes, pero otros no. Por esto, sus habitantes no desarrollan un sentimiento de pertenencia hacia estas construcciones. Para poner a prueba esta hipótesis, se definieron criterios de diseño adaptados de Díaz (2015), arquitecto y autor de origen Pewenche que entrega análisis de la cultura y los modos de vida Pewenche para un seminario de la carrera de 
Arquitectura. Adicionalmente, se adaptaron los perfiles arquitectónicos extraído desde la "Guía de diseño arquitectónico Mapuche" (Ministerio de Obras Públicas [MOP], 2016), la cual brinda una perspectiva centrada en el diseño simbólico, el territorio e impacto social que conllevó la construcción de cada edificio público allí estudiado.

\section{METODOLOGÍA}

Dada la casi total inexistencia de estudios respecto a los niveles de pertenencia de la población indígena hacia edificios en áreas urbanas, el enfoque de esta investigación es de carácter cualitativo exploratorio. Así, se analizan los criterios de diseño Pewenche identificados a partir de Díaz (2015) y MOP (2016), los cuales incluyen orientación, morfología, adaptación al clima, adaptación por medio de la materialidad y participación ciudadana en el proceso de diseño. Las técnicas de recolección de datos seleccionadas son el análisis planimétrico urbano y bibliográficos, de ambos casos de estudio, mediante el cual se obtienen datos relevantes con respecto a su emplazamiento, su relación con el contexto y participación. La observación arquitectónica, ocupando instrumentos como la fotografía y el croquis, permitieron generar análisis acerca del criterio de adaptación al territorio que aplica el edificio público: cómo responde al clima y a su entorno natural mediante su formalidad y materialidad. Los datos respecto a la participación en el proceso de diseño de los edificios fueron consultados a la Dirección de Obras Municipales de Alto Bio Bío.

Se seleccionaron dos edificios públicos representativos en la localidad de Ralco, arrojados en una encuesta de "Satisfacción ciudadana", el año 2017, realizada por la Municipalidad de Alto Biobío en conjunto a la Dirección de Obras Municipales (DOM). Esta encuesta -que tuvo la finalidad de obtener datos enfocadas en aspectos laborales, sociales, económicos y culturales de la comuna- involucró a 1.200 habitantes, de los cuales el 85\% se declaró Pewenche y el 15\% no Pewenche. También definió que un 48\% de los encuestados pertenece a la zona urbana y el $52 \%$ a comunidades rurales. Dentro de la encuestas, se consultaron aspectos socioculturales incluyendo preguntas sobre el nivel de pertenencia cultural de los edificios públicos ubicados en Ralco ${ }^{1}$. Se consultó cuáles son los edificios públicos con mayor pertenencia cultural y aquellos de mayor relevancia para la comunidad, en opinión de los residentes. Dentro de los resultados obtenidos por la encuesta, se destaca que el $48 \%$ de la población declaró no sentir pertenecía con los edificios públicos municipales y el 33\% no los reconoció como arquitectura con pertenencia. Estos resultados indican que más del $80 \%$ de la población se encuentra insatisfecha con sus edificios públicos representativos.

${ }^{1}$ Es importante señalar que la encuesta no tuvo un sustento teórico especifico, sino que consulta por el sentido común de la palabra "pertenencia cultural" para los residentes urbanos y no urbanos de la comuna. 
1. Un $67 \%$ de la población encuestada, indicia el Museo Pehuenche como el equipamiento con mayor pertenencia e identidad cultural Pehuenche; seguido por el edificio Municipal, con un 23\% y el liceo técnico multicultural y biblioteca municipal, ambos con una preferencia del $5 \%$.

2. Un $83 \%$ de la población encuestada, reconoció el edificio Municipal como el equipamiento público de mayor relevancia para la localidad de Ralco. Seguido por el CESFAM, con un 10\%, y el liceo técnico multicultural, con un $7 \%$.

De esta manera, el Museo Pehuenche es considerado el edificio con mayor pertenencia cultural y el edificio municipal fue seleccionado como el equipamiento de mayor relevancia para la comunidad. Ambos edificios, por lo tanto, son reconocidos públicamente por la comunidad, por lo cual son casos interesantes para ser evaluados en esta investigación.

Por un lado, el Museo Pehuenche fue construido el año 2010 por la oficina de arquitectura Víctor Lobos Ltda. Cuenta con una superficie de 192 m2 y una torre/mirador de 18 metros de altura. Por otro lado, el edificio consistorial Municipal de Alto Biobío fue proyectado el año 2010, por el arquitecto Saúl Navarrete Paredes. Cuenta con aproximadamente 1.500 m2 y con una inversión de \$1.500.000.000 en su ejecución.

\section{MARCO TEÓRICO}

\section{Creación de identidad en centros urbanos}

La problemática más amplia abordada en el presente artículo giró en torno a cómo crear un sentimiento de identidad para las poblaciones indígenas en áreas urbanas. El pueblo Mapuche, en general, y la cultural Pehuenche, en particular, se ha caracterizado por ciertos elementos identitarios adscritos a las zonas rurales del país. Pero, con la migración masiva a ciudades, hoy constituye un desafío el crear espacios y edificios públicos que contribuyan al sentimiento de pertenencia de las poblaciones indígenas en las ciudades (Huencho, 2016).

En esta investigación, se reconoce al pueblo Mapuche como originario del territorio a partir de la costa hasta la cordillera abarcando desde el valle central hacia el sur de Chile, terminando en la actual isla de Chiloé. Esta extensión del pueblo Mapuche se divide en tres grandes identidades territoriales: Lafkenche, en la costa; Wenteche, en el valle y Pewenche, en la cordillera (Chihuailaf, 1999). Se establecen bajo la misma cosmovisión Mapuche, pero cada uno con sus propios modos de vida adaptados al territorio que habitan, lo que proporciona condiciones y criterios de diseños arquitectónicos característicos en cada uno de ellos.

La cultura Pewenche, dada su ubicación geográfica, se configura en dos espacios económicos importantes ligados por los senderos (Huiliñir, 2015). Por un lado, la invernada, que es la temporada de invierno donde -junto al ganado- bajan a los planos geográficos del territorio y las veranadas que, 
por su parte, son las temporadas de verano, cuando suben para alimentar y comercializar el ganado a la precordillera. Estos senderos son los que construyen, entonces, la identidad territorial Pewenche (Huiliñir, 2015). Si bien la arquitectura tradicional Pewenche recoge criterios de diseño generales de la cosmovisión Mapuche como orientación al Puel Mapu y morfología circular centralizada, la adaptación al territorio cordillerano le entrega una condición única. Entre lo que se destaca la altura y pendiente de su sistema de techumbre como respuesta al clima extremo (lluvias y nevazones), la utilización de la piedra como materialidad principal para la absorción del calor durante el día y la liberación de este durante las frías noches y adaptación a pendientes rocosas (MOP, 2016).

El centro urbano de Ralco aparece dentro de ese territorio histórico como espacio intercultural y adicionado, donde la pertenencia de los edificios y espacios públicos aún está por construirse. En ese sentido, es importante entender que el territorio es una construcción sociopolítica, expresión de una red de relaciones de poder, que permite la formación de identidades (Caniguan, 2020). Más puntualmente, las identidades territoriales sirven para excluir e incluir, para validar ciertas autoridades por sobres otras. De esa manera, los edificios públicos en los espacios urbanos son fundamentales, porque los "territorios se componen de hitos geográficos y simbólicos y de un centro, vistos como densificaciones significativas ... con poder de convocatoria y socialmente emblemáticos" (Caniguan, 2020, p. 23). Más que en las áreas rurales, los edificios tienen un potencial para generar un entendimiento del territorio urbano a partir de su vocación intercultural, ya sea como lugar mapuche o como lugar no-mapuche.

En respuesta a la hipótesis planteada de la carencia de identidad y la necesidad de reinterpretación de las culturas indígenas en los edificios públicos de las ciudades chilenas, este estudio analiza la relación entre la aplicación de los criterios de diseño arquitectónico indígena y el sentimiento de pertenencia real percibido ex-post, o sea, después de construido los edificios y vivenciados por la población por un período de algunos años.

La Guía de diseño arquitectónico Mapuche/Aymará para edificios yespacios públicos, publicado por el MOP originalmente en el año 2003 y reeditado en 2016, es una guía que postula criterios de diseño a ser aplicados en edificios públicos para crear pertenencia cultural. Sin embargo, dado que no existen estudios para corroborar si esos lineamientos se traducen en edificios reconocidos por la población como de mayor pertenencia, surge la necesidad urgente de analizar la aplicación de los lineamientos para evidenciar si las decisiones a tomar en la proyección de un edificio público llevan a edificios con mayor pertenencia cultural a relacionarse con los pueblos indigenas.

Para eso planteamos la pregunta: ¿Dentro de la arquitectura pública en Ralco, la aplicación de criterios de diseño pertinentes lleva a que los edificios exhiban pertenencia cultural?

\section{Criterios de diseño arquitectónico Mapuche/Pewenche}

Los criterios presentados en esta investigación se adaptan de los postulados por Díaz (2015). En primer lugar, existen dos criterios de carácter 
general: orientación y morfología, los cuales se vinculan directamente con el Pueblo Mapuche y su cosmovisión de forma transversal a lo largo de todo su territorio. En segundo lugar, se recogen los postulados presentados por el MOP (2016), los cuales destacan criterios específicos que derivan de las características propias del territorio cultural Pewenche, nos referimos a los criterios de: adaptación al clima y materialidad, agregado por último a partir de MOP (2016) el criterio de: participación ciudadana, en el proceso de diseño.

\section{Orientación}

El territorio ancestral pewenche es concebido como totalidad bajo la estructura de relaciones meli witxan mapu, el encuentro de las cuatro tierras: Puel Mapu, la tierra del este; Pikun Mapu, la tierra del norte; Lhafken Mapu, la tierra del mar; Willi mapu, la tierra del sur. (Navarrete, 2017). Por esto, la entrada de la ruka mira en dirección al este o Puel Mapu, que es el lugar donde moran las fuerzas espirituales benéficas, tornando su vida hacia la salida del sol entre las mawida (montañas), donde crece el Pehuen (araucaria), árbol sagrado que le entrega vida e identidad al pueblo.

\section{Morfología}

Las morfologías de las construcciones Pewenche derivan de sus espacios ceremoniales (por ejemplo, nguillatun). Estas se establecen mediante una geometría circular, soportada estructuralmente solo por pilares que generan espacios permeables, factor sumamente importante y relevante a considerar al momento de proyectar su arquitectura. Esta geometría centralizada representa el wenu mapu o espacio sagrado, que es el lugar donde habitan los antepasados, que no se direcciona hacia el exterior, sino que se cierra o abre hacia su interior, aglomerando y enfocando todas sus vistas, su atención y espacios en lo que ocurre en torno al kutral (fuego), que representa el sol y que congrega el encuentro (Díaz, 2015). La centralidad -y el círculo- es vista como un espacio amplio, que abraza y acoge, no jerarquiza e iguala condiciones proxémicas para quienes lo habitan.

\section{Adaptación al Clima}

En los sectores más bajos de la precordillera, predomina el clima mediterráneo. La zona mantiene una temperatura entre los 10 oc y 20 o-C, con su máxima en verano. Las lluvias alcanzan un promedio de 1.440 $\mathrm{mm}$ al año, con un máximo de $240 \mathrm{~mm}$ mensuales y sobre los $800 \mathrm{msnm}$. Además, se registran fuertes nevazones en época invernal (MOP, 2016). Este clima condiciona el diseño arquitectónico de los pobladores, ya que proyectan sus cubiertas en respuesta a las condiciones del clima, ampliando sus pendientes hasta $60^{\circ}$; aumentando sus alturas por sobre los 3 metros, en algunos casos; cubriendo circulaciones exteriores e interiores, sus espacios públicos y ceremoniales e, incluso, aplicando sistemas constructivos propios como las canogas (Figura 1), que permiten un mejor escurrimiento de aguas lluvias y aguas nieves (MOP, 2016). 
Figura 1

Amarre en "canogas", en Casa de la Cultura Pewenche.

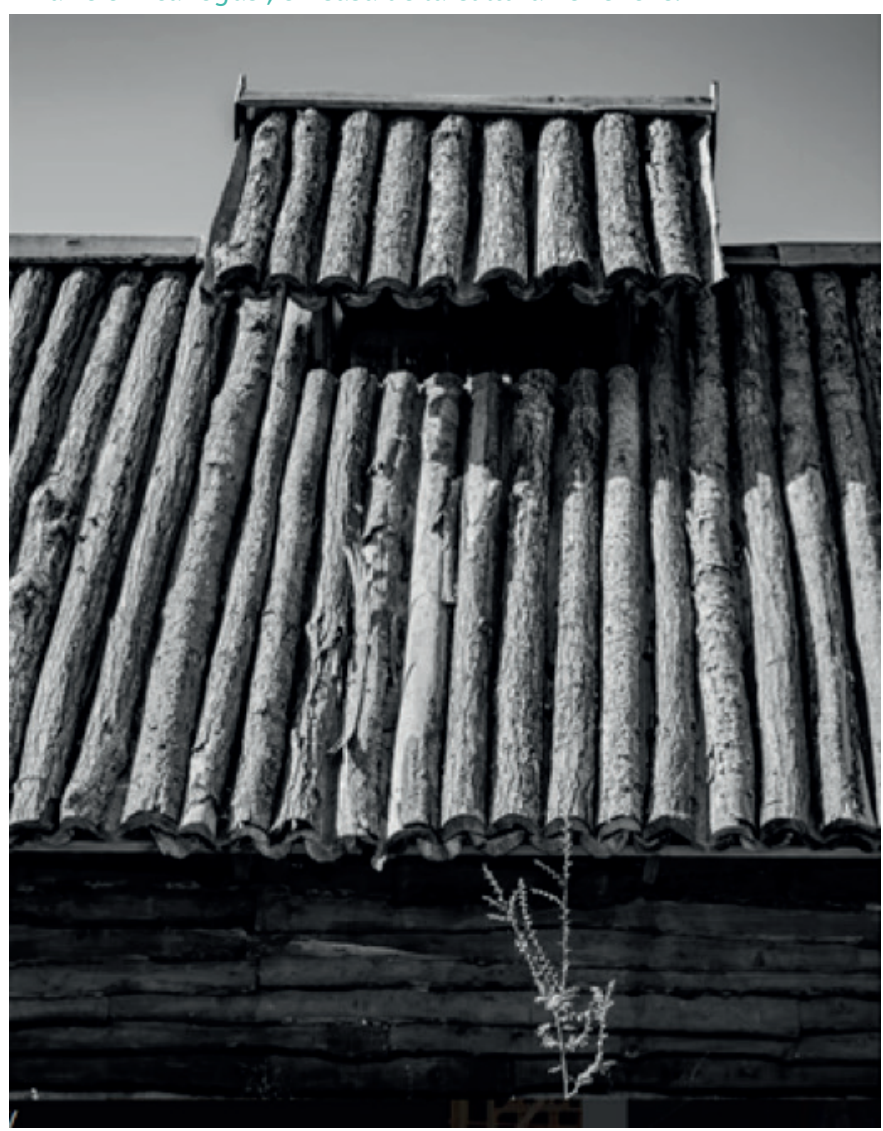

Fuente: Franco Romero

\section{Materialidad}

Las viviendas y los espacios colectivos Pewenche tienen una excelente respuesta a las condiciones del clima, una buena resistencia y fácil manipulación. Claro ejemplo es la ruka, un sistema de construcción que se adapta al territorio y que involucra la madera, originalmente nativa, como soporte estructural. Por último, la piedra no es utilizada como elemento estructural, sino que en pircas, cercos y muros (MOP, 2016). Por último, la piedra es utilizada como un recurso de regulación de temperatura natural, absorbiendo el calor del sol durante el día y liberándolo por las noches. Se encuentra en pircas, cercos y muros, pero no como elemento estructural (MOP, 2016).

\section{Participación}

Finalmente, el quinto criterio de la participación ciudadana en el proceso de diseño es fundamental para evitar que la aplicación de los criterios anteriores no sea mera aplicación estética vaciada de sentido, como acontece en muchos procesos multiculturales donde se espera que la cultura indigena no interfiera en la cultura (urbana) dominante (Santos, 2012). Por eso, aquí se entiende el proceso de creación de pertenencia a través de la arquitectura como un proceso intercultural, dinámico y no unidireccional. 


\section{RESULTADOS}

\section{Análisis de la arquitectura pública}

\section{Edificio Público Municipal de Alto Biobío}

Orientación en la Figura 2 se puede apreciar el emplazamiento del edificio Municipal, el cual nos permite determinar tres factores importantes. Primero, el edificio se encuentra orientado hacia el Puel Mapu, brindando un marcado acceso semicircular que recibe al sol por las mañanas. El edificio entiende el espacio del acceso orientado al este, como un recinto principal y de suma importancia dentro del diseño arquitectónico Pewenche, donde se aprecia como el acceso también se convierte en un amplio espacio público, de encuentro e intercambio cultural, al cual se le destinan roles administrativos relevantes, reconociendo así, la jerarquía de un espacio, que destaca en el diseño Pewenche por sobre otros.

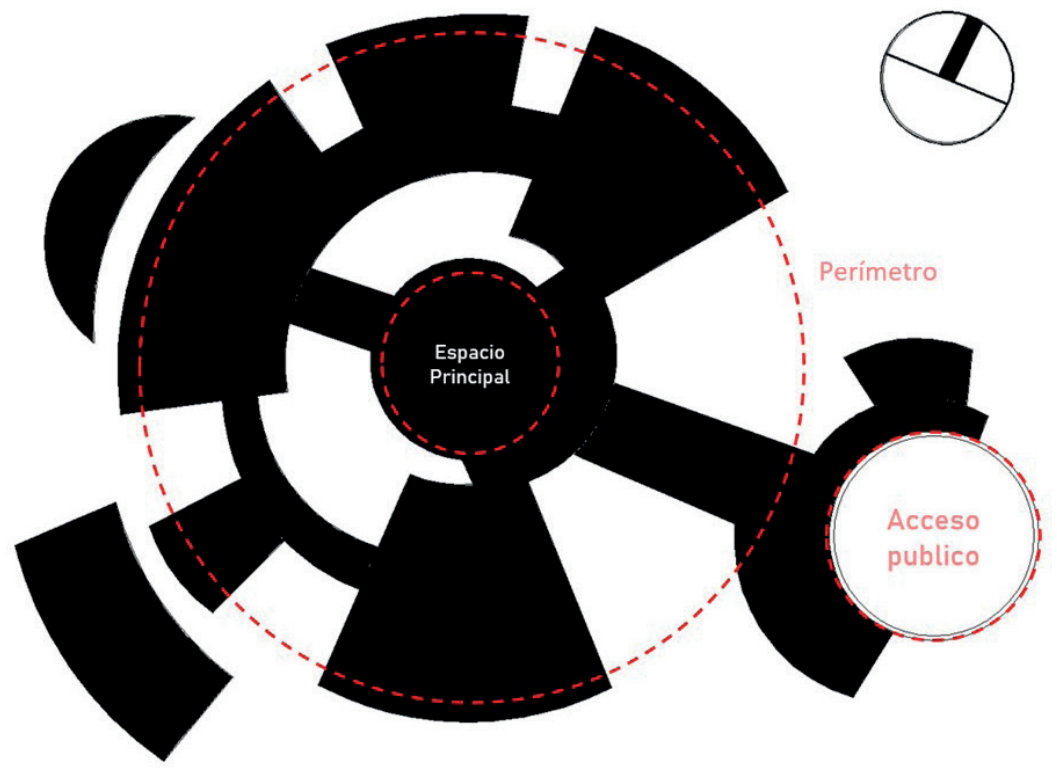

Fuente: Elaboración propia.

Morfología El gran espacio de recepción que recibe al público adquiere la forma de una ruka pewenche, que se eleva unos 12 a 15 metros. En su base incorpora un fogón en obra para albergar el kutral, fuego, en torno al cual se desarrolla la oralidad y socialización de los usuarios.

La luz natural es captada al interior cenitalmente, en la presencia del fogón. Por su parte, el espacio profano, por cuanto alberga las diferentes dependencias en que se organiza la municipalidad, presenta una tipología espacial funcional acorde al programa que acoge, con diversas oficinas alineadas a través de un pasillo circular que las organiza de forma radial 
al espacio central. Tal ordenamiento evoca la disposición de las ramadas, en el nguillatun, que se distribuyen semicircularmente orientadas al Este, permitiendo articular la actividad tradicional con la doméstica o funcional del municipio (Figura 3).

La geometría circular del espacio central del edificio acoge usos y hábitos propios de la cultura Pewenche, al disponer en su perímetro de bancas donde la comunidad puede socializar a la espera de atención. La disposición de este mobiliario permite que todos se miren a la cara mientras están sentados, estableciéndose, así, relaciones horizontales entre las personas.

\section{Figura 3}

Acceso Principal del edificio público Municipal orientado hacia el Puel Mapu.

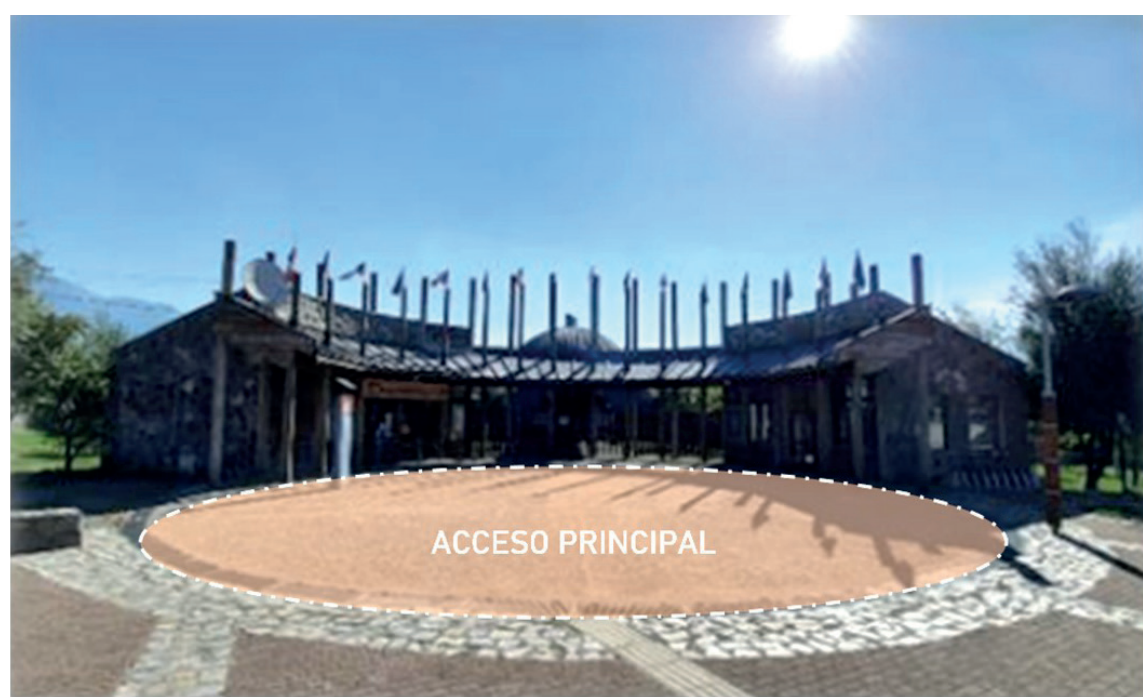

Fuente: Elaboración propia.

Clima La respuesta al clima se evidencia en un tipo de cubierta con alturas mayores a los 2 metros, de formas curvas y de tonalidades negras, las que ayudan a un mejor deslizamiento de las aguas lluvias y derretimiento de aguas nieves. También contempla un segundo tipo de cubierta, en formatos planos de un agua con pendientes pronunciadas que genera un fácil y mayor deslizamiento de aguas lluvias y nieve.

Materialidad Los materiales predominantes en la obra corresponden a madera y piedra, ambos presentes de manera abundante en el territorio. En este sentido, su uso en el edificio constituye una reinterpretación material del uso tradicional. Por su parte, las soluciones y técnicas constructivas no presentan características asociadas a la cultura local, sino más bien a desarrollos tecnológicos modernos. El sistema constructivo aplicado en el espacio central se basa en vigas de madera laminada (Figura 4) y cerramientos en madera. En cuanto al anillo exterior de las dependencias municipales, se estructura a partir de muros de hormigón armado y tabiques de madera (Figura 5). En algunas zonas se observan revestimientos en piedra de cantera. 
Figura 4

Fogón y recepción principal de la Municipalidad.

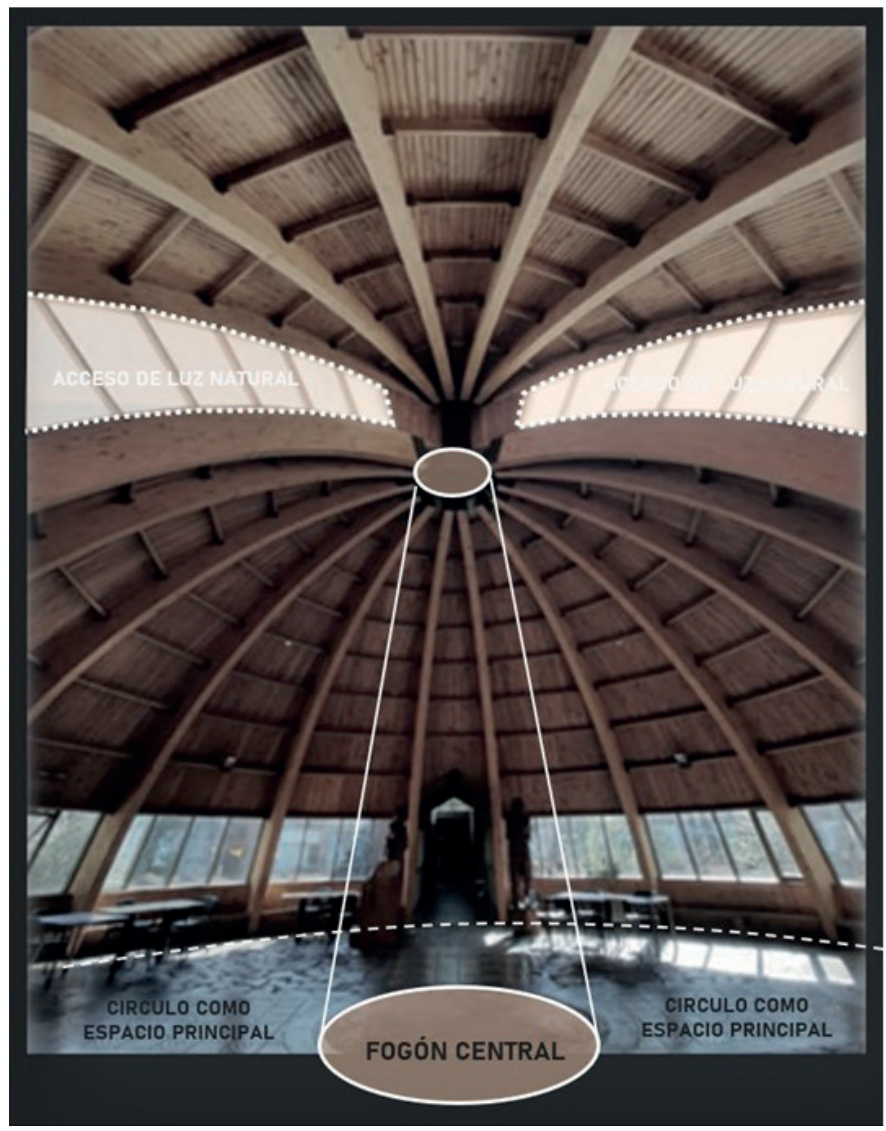

Fuente: Elaboración propia.

Figura 5

Materialidad del edificio público en base a la madera y la piedra.

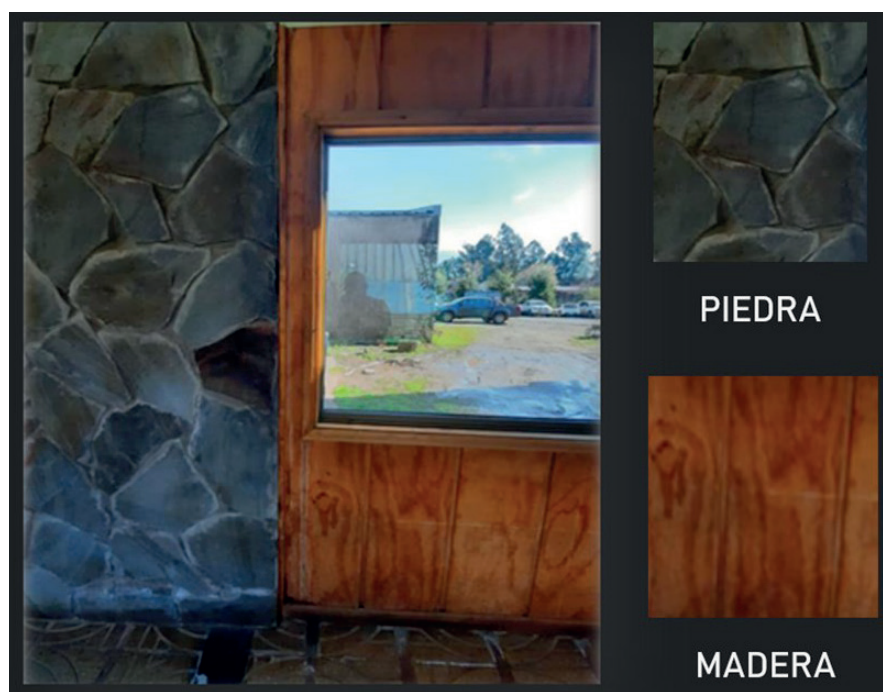

Fuente: Elaboración propia. 
Participación Este edificio no contó con un proceso de consulta ciudadana, pese a ser construido con dineros estatales, esto debido a que la municipalidad fue el primer equipamiento público del lugar y no se encontraba una población urbana establecida que pudiera desarrollar aquel proceso. El arquitecto Pewenche, Saul Navarrete, aplicó conocimientos propios, tanto técnicos como socioculturales, en el proceso de diseño del edificio municipal, motivo por el cual podemos asociar su alto nivel de pertenencia cultural.

\section{Museo Pewenche.}

Orientación El Museo Pehuenche se encuentra orientado hacia el oriente, mirando el Puel Mapu, recibiendo energía del sol por las mañanas (Figura 6) y aplicando correctamente este criterio Pewenche con respecto a la ubicación de su acceso. El edificio entrega un considerable espacio para la realización de actividades públicas en su acceso, brindándole una zona de anfiteatro, de paseo peatonal, de encuentro y de intercambio comercial. Todo esto orientado hacia el este, respetando y reconociendo la importancia de este espacio en la cultura ancestral Pewenche.
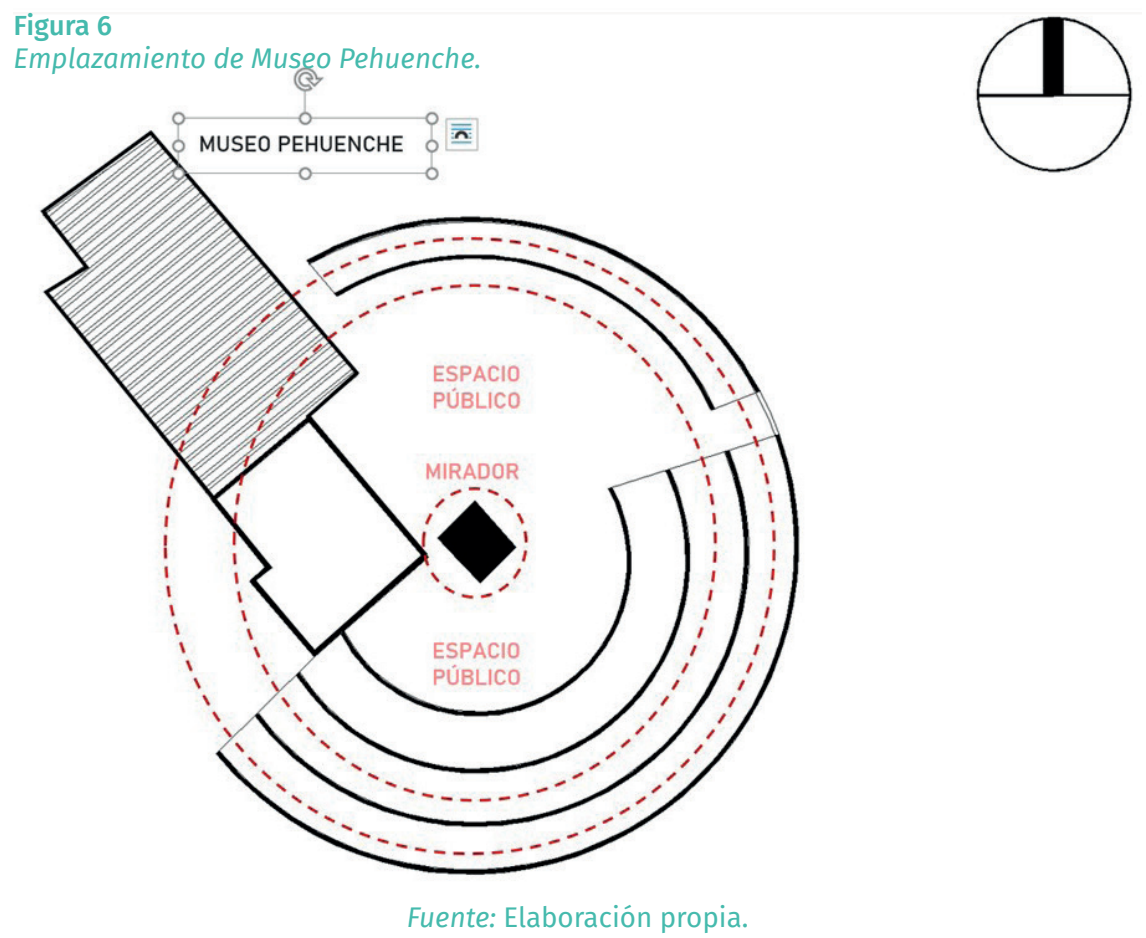

Morfología Este equipamiento presenta una volumetría con geometría paralelepipeidal, la cual es dividida en 3 sectores, la administrativa, de recepción y de exposición, centrando su atención en este último, provocando la ausencia de un centro que recojan las actividades principales e individualizando cada espacio.

Este proyecto desarrolla un trabajo mayor en su acceso, en el cual, morfológicamente, se basa en los criterios de diseño arquitectónico Pewenche, 
generando estos espacios públicos circular, centralizando las actividades principales mediante el desarrollo de un mirador que permite la conexión inmediata con su contexto.

Clima La respuesta al clima, mediante el diseño del edificio, la soluciona a través de la aplicación de una cubierta a un agua, con una pendiente inferior a los $30^{\circ}$, que, si mantiene tonalidades oscuras para un rápido derretimiento de aguas nieves, pero carece de identidad cultural.

El Museo Pehuenche maneja la entrada de luz natural mediante grandes ventanales orientados al norte y bloquea la entrada de luz y calor desde el oriente, proyectando un gran muro de piedra que no le permite su paso.

En cuanto al mirador ubicado en el acceso, es cubierto y semi cerrado, generando una sensación de refugio que ampara del clima, permitiendo la entrada de luz y ventilación mediante su circulación vertical.

Materialidad La materialidad seleccionada para la construcción de este equipamiento público se basa en madera, piedra y acero (Figura 7). Presenta un sistema constructivo metálico, el cual es revestido en gran parte por la piedra, generando con esto una sensación de mayor nexo con lo natural mediante el material y dejando la madera como tercer material del proyecto, ocupada principalmente en el mobiliario interior y las estructuras desarrolladas en el espacio público que genera su acceso.

\section{Figura 7}

Acceso principal del edificio público del Museo Pehuenche.

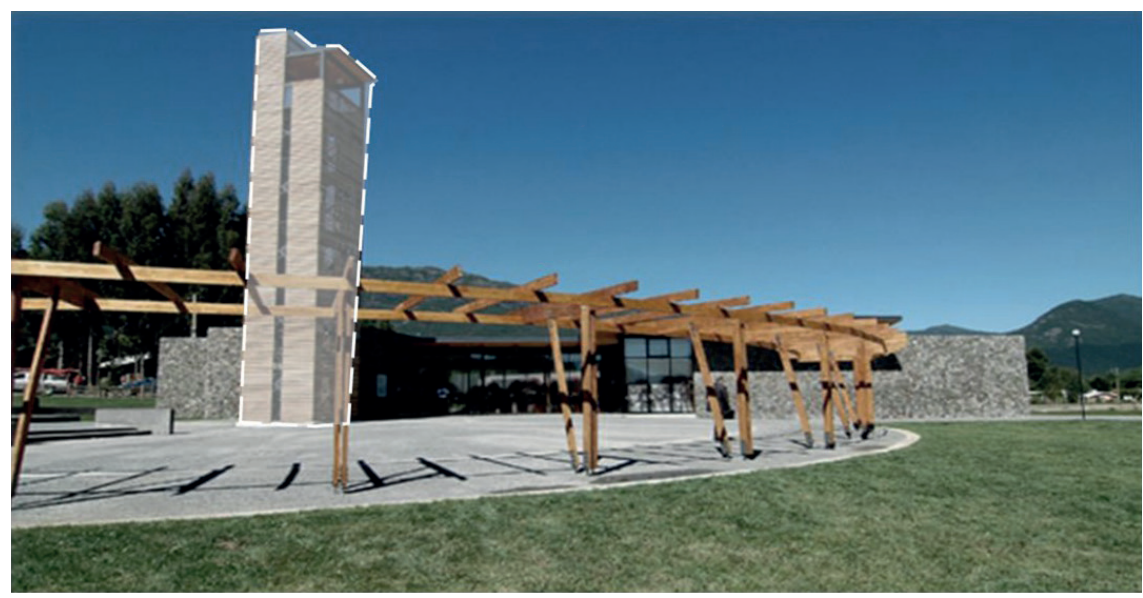

Fuente: Elaboración propia.

Participación La construcción del museo tampoco contó con un proceso de consulta ciudadana, habiendo sido construido por un estudio de arquitectura privado dirigido por el arquitecto Víctor Lobos. La oficina no pertenece a -ni presenta vínculos culturales con- la zona, lo que se vio reflejado en el estudio donde este edificio público arrojó un bajo nivel de pertinencia cultural.

Los resultados del análisis se resumen a continuación (Tabla 1) en conjunto con los resultados de la encuesta. 
Tabla 1

Aplicación de los criterios de diseño en contraste a la encuesta aplicada.

\begin{tabular}{llcc} 
& & MUSEO PEHUENCHE & EDIFICIO MUNICIPAL \\
\hline CRITERIOS DE DISEÑO & Orientación & $\mathrm{Si}$ & $\mathrm{Si}$ \\
\hline & Morfología & $\mathrm{No}$ & $\mathrm{Si}$ \\
\hline & Adaptación al clima & $\mathrm{No}$ & $\mathrm{Si}$ \\
\hline & Materialidad & $\mathrm{No}$ & $\mathrm{Si}$ \\
\hline & Participación & $\mathrm{No}$ & $\mathrm{No}$ \\
\hline & & & $\mathrm{Si}$ \\
\hline DATOS DE ENCUESTA & Mayor relevancia & No & No \\
\hline & Mayor pertenecía & $\mathrm{Si}$ &
\end{tabular}

Fuente: Elaboración propia.

Ambas muestras de estudio demostraron, según la evaluación de los criterios utilizados, un nivel de pertinencia y pertenencia cultural que se contrarrestaba. Esto se aprecia en el Museo Pehuenche, que presenta un $25 \%$ de pertinencia, en contraste a un $67 \%$ de pertenencia, y el edificio Municipal, un $100 \%$ de pertinencia, pero solo un $23 \%$ de pertenencia, lo que nos permite responder nuestra hipótesis planteada. Los edificios públicos analizados recogen solo ciertos criterios de diseño Pewenche, lo que no permite generar un sentimiento de pertenencia hacia estos. Sin embargo, podemos comenzar a concluir que el bajo desarrollo del sentido de pertenencia hacia los edificios públicos ubicados en Ralco no deriva solo de una inadecuada pertinencia en su diseño arquitectónico, sino que por otro factor que logramos identificar, que es la baja valorización que se le entrega al diseño de los espacios más relevantes de los proyectos. Por ejemplo, el acceso en su exterior y el principal en su interior, ya que son esos lugares los más apreciados dentro de la cultura Pewenche porque promueven la interculturalidad, el intercambio de saberes, costumbres y modos de vida de diversas culturas que allí se encuentran.

Se resume el análisis de los niveles de pertinencia y de pertenencia a continuación (Tabla 2).

Tabla 2

Resultado de niveles de pertenencia y pertinencia.

\begin{tabular}{lll}
\hline NIVEL DE PERTINENCIA & $25 \%$ & $100 \%$ \\
\hline NIVEL DE PERTENENCIA & $67 \%$ & $23 \%$ \\
\hline
\end{tabular}

Fuente: Elaboración propia.

\section{DISCUSIÓN Y CONCLUSIONES}

En resumen, este estudio plantea el análisis de dos edificios públicos más destacados por la comunidad de Ralco, mediante criterios postulado por Díaz (2015) y MOP (2016), tanto generales (orientación y morfología), como también criterios específicos (adaptación al clima y materialidad) entregados por los modos de vida adoptados en el territorio cordillerano, y finalmente de la participación. Seguido de esto, se trabaja con la 
encuesta Satisfacción ciudadana realizada por la Municipalidad junto a la Dirección de Obras Municipales, en las cuales se genera un contraste con respecto a los resultados obtenidos en el primer análisis, por lo que se postula este sentido de pertenencia, de arraigo, priorizando lo que entrega el proyecto culturalmente por sobre lo que arquitectónicamente representa.

Esta investigación permite dar respuesta al cuestionamiento planteado al comienzo del estudio, sobre la existencia de edificio públicos representativos culturalmente en la localidad de Ralco. Los resultados obtenidos muestran que el Museo Pehuenche, por un lado, califica con un bajo nivel de pertinencia en cuanto a los criterios seleccionados en su diseño arquitectónico. Sin embargo, desarrolla el mayor sentido de pertenencia por parte de la comunidad mediante sus espacios de encuentro, de intercambio cultural y expositivo, lo que genera esa identidad. Por otro lado, el edificio municipal de Alto Biobío se identificó como el de mayor pertinencia en cuanto al análisis de los criterios de diseño arquitectónicos estudiados. También, es reconocido como el de mayor importancia o relevancia de la localidad de Ralco, pero, pese a eso, carece de la apropiación y sentimiento de arraigo por parte de la comunidad producto de que no integra socialmente la cultura, solo la reinterpreta de manera arquitectónica mediante su estructura.

Por último, esta investigación busca relacionar la aplicación de los criterios pertinentes y la generación de una real pertenencia cultural Pehuenche con los edificios públicos situados en la localidad de Ralco. Al respecto, se pudo evidenciar que el concepto de pertenencia no se puede generar de manera automática, ni siquiera un diseño pertinente culturalmente logra asegurar el desarrollo emotivo de pertenencia. No existe un manual o guía que permita garantizarla (Huencho, 2016), sino más bien herramientas que ayudan y encaminan la búsqueda de ésta. Esta investigación recoge lo planteado por Toro (2017), sobre la importancia de cambiar el rol de los profesionales de arquitectura desde el de creador exclusivo a uno donde prime la co-creación con las comunidades. Justamente, en ambos casos no se dio un proceso de participación ciudadana en el diseño, lo que quizás pudo haber sido fundamental para generar mayor pertenencia respecto a los edificios.

Este estudio deja abiertas nuevas interrogantes a considerar en investigaciones futuras, focalizadas en el desarrollo de la pertenencia para estas nuevas construcciones públicas que están surgiendo en territorios con una alta identidad cultural y que buscan el mayor nivel de representatividad en estos. Es importante realizar investigaciones con una muestra que incluya mayor número de edificios, en espacios urbanos ubicados en otras identidades territoriales mapuche, en territorios de otros pueblos (Aymara, Rapa Nui, etc.).

Pero, por sobre todo, se hace necesario investigar edificios que cuenten con participación ciudadana. Esa fue una carencia encontrada en el actual estudio y creemos sumamente necesaria en la construcción de pertenencia, pertinencia e identidad, tanto con el territorio como con quienes lo habitan y el fortalecimiento de la interculturalidad en el ámbito de la edificación pública. Esto es esencial en casos como Ralco, donde los 
edificios públicos son los responsables de crear una pertenencia cultural en un área urbana inserta en un territorio indigena que históricamente ha sido despojado de tierras e identidad. Finalmente, están las nuevas variables de diseño que nos referíamos en el análisis, en torno a los espacios de acceso que proponen los proyectos, al tipo de actividad (programa) que promueven, independiente de su rol administrativo, el diseño de sus espacios principales y accesibilidad, todos estos factores encontrados durante esta investigación que pueden promover un mejor desarrollo de la pertenencia en estos edificios de equipamiento público en áreas urbanas interculturales.

\section{REFERENCIAS BIBLIOGRÁFICAS}

Araos, V. (2012). Presentación del nuevo equipamiento público en la comuna de Alto Biobío. Diario La tribuna, p. 8.

Caniguan, N. (2020). Construcciones sociopolíticas del territorio. Movimientos indígenas y políticas públicas, la configuración de los espacios locales. Revista CUHSO, 30(2), 19-40.

Chihuailaf, E. (1999). Recado confidencial a los chilenos. LOM Ediciones.

Díaz, Y. (2015). Construcción paisaje cultural Pehuenche. Facultad de arquitectura, Universidad de Chile.

Dirección de Obras Públicas. (2017). Encuesta de satisfacción ciudadana en el área urbana de la comuna de Alto Biobío. Insumos para el Plan Regulador Comunal de Alto Biobío. Documento no publicado.

Huencho, E. (2016). Espacio público e identidad mapuche urbana. En: W. Imilán (Ed.), Pueblos originarios y hábitat residencial urbano: diálogo sobre políticas habitacionales y pertinencia cultural en Chile (pp. 66-71). CONADI, INVI.

Huiliñir, V. (2015). Los senderos Pehuenche en Alto Biobío (Chile): articulación espacial, movilidad y territorialidad. Revista de Geografía Norte Grande, (62), 47-66.

Instituto Nacional de Estadísticas, INE (2018). Sintesis de resultados censo 2017.

Ley N¹9.959. Creación de la comuna de Alto Biobío, en la región del Biobío, Congreso nacional, Santiago, 21 de Julio, 2004.

Ministerio de Obras Públicas. (2016). Guía de diseño arquitectónico Mapuche/Aymará para edificios y espacios públicos. Coordinación editorial MOP.

Navarrete, L. (2017). Ruka: Representación arquitectónica y simbólica del mundo Mapuche. CONADI. 
Plan de Desarrollo Comunal. (2014). Subdivisiones. Ralco, Alto Biobío. http://www.transparenciaaltobiobio.cl/

Santos, B. S. (2012). Cuando los excluidos tienen Derecho: justicia indigena, plurinacionalidad e interculturalidad. En B. Santos y A. Jiménez (Eds.), Justicia indígena, plurinacionalidad e interculturalidad en Ecuador (pp. 13-50). Abya Yala/Fundación Rosa Luxemburg.

Toro, R. (2017). Nuevo rol del arquitecto creador al arquitecto ciudadano. Diseño participativo en la producción del hábitat En: Imilan, W., Larenas, J., Carrasco, G., y Rivera, S. (Eds.), ¿Hacia dónde va la vivienda en Chile?: nuevos desafíos en el hábitat residencial (pp. 109-118) Editorial Adrede. http://repositorio.uchile.cl/handle/2250/144898

Toro, A. [Muñoz D.] (2013). Documental "Berta y Nicolasa, las Hermanas Quintreman" [Archivo de Video]. Youtube. https:// www.youtube.com/watch?v=thVAb8wE9AQ\&t=6s 